\title{
Passive vibration on the legs reduces peripheral and systemic arterial stiffness
}

\author{
Hypertension Research (2012) 35, 126-127; doi:10.1038/hr.2011.164; published online 13 October 2011
}

Intermittent leg exercise $(10 \times 1$-min sets $)$ with whole-body vibration (WBV) decreases brachial-ankle pulse-wave velocity $(\text { baPWV })^{1}$ and leg PWV (legPWV) but not aortic PWV. ${ }^{2}$ As baPWV is an index of systemic arterial stiffness $^{3}$ mainly influenced by aortic PWV $(\sim 58 \%)$ and legPWV $(\sim 23 \%),{ }^{4}$ previously published results ${ }^{1,2}$ suggest that WBV affects baPWV through peripheral but not central PWV. The post-exercise decrease in PWV is associated with vasodilation in the exercised limb. ${ }^{5,6}$ Similarly, intermittent WBV $(3 \times 3$ min sets $)^{7}$ or passive vibration $(\mathrm{PV})^{8}$ has been shown to increase blood flow in the vibrated limb after only 1-min post vibration. Interestingly, 10 min of continuous PV was found to increase arm skin blood flow after 5-min post vibration, ${ }^{9}$ indicating a direct relationship between the duration of exposure and vasorelaxation. We hypothesized that PV on the legs may decrease legPWV and baPWV more than aortic PWV. The purpose of our study was to examine PWV responses following continuous PV of lengthy duration.

A total of $23(\mathrm{M}=10$ and $\mathrm{F}=13)$ healthy (age $=23 \pm 3$ years, height $=1.67 \pm 0.02 \mathrm{~m}$ and weight $=67.3 \pm 3.5 \mathrm{~kg}$ ) subjects provided their written consent, which was approved by the Institutional Review Board. The no-vibration control (CON) and PV sessions were randomized and separated by $48-72 \mathrm{~h}$. Subjects rested in the supine position with their thighs and calves over the vibration platform (Powerplate, Badhoevedorp, The Netherlands) for $20 \mathrm{~min}$ before testing, $10 \mathrm{~min}$ of CON or PV, and $30 \mathrm{~min}$ of recovery. Their legs were directly exposed to continuous vibration at a frequency $(25 \mathrm{~Hz})$ and amplitude $(2 \mathrm{~mm})$ that provided an acceleration of $\sim 5.37 \mathrm{G}$ based on studies showing increased blood flow ${ }^{8,10}$ or decreased baPWV ${ }^{1}$ and legPWV $\mathrm{V}^{2}$ following intermittent or continuous vibration of similar total duration and/or intensity.
PWV and brachial blood pressure (BP) were measured in duplicate with an automatic device (VP-2000, Omron Healthcare, Vernon Hills, IL, USA), which uses BP cuffs around both arms (brachial artery), and ankles (posterior-tibial artery) and tonometers over the right carotid and femoral arteries to obtain baPWV, aortic PWV (carotid-femoral) and legPWV (femoral-ankle). The feet of the pulse waves were related to the ECG's R-wave to calculate transit time. The distance between sampling points for aortic PWV was measured with a non-elastic tape, whereas for baPWV and legPWV, this value was calculated according to the subject's height. ${ }^{3}$ Path lengths from the suprasternal notch to the brachial, femoral and tibial arteries, and from the femoral to the tibial

Table 1 BP, HR and aortic PWV before and after 10-min continuous control and passive vibration bouts

\begin{tabular}{|c|c|c|c|c|}
\hline & Baseline & Post-3 & Post-15 & Post-30 \\
\hline \multicolumn{5}{|c|}{$S B P(m m ~ H g)$} \\
\hline Control & $114 \pm 2$ & $114 \pm 2$ & $113 \pm 2$ & $113 \pm 2$ \\
\hline PV & $112 \pm 2$ & $114 \pm 2$ & $113 \pm 2$ & $114 \pm 2$ \\
\hline \multicolumn{5}{|c|}{$D B P(m m ~ H g)$} \\
\hline Control & $64 \pm 1$ & $64 \pm 1$ & $63 \pm 1$ & $64 \pm 1$ \\
\hline PV & $62 \pm 1$ & $64 \pm 1$ & $63 \pm 1$ & $64 \pm 1$ \\
\hline \multicolumn{5}{|c|}{$M A P(m m ~ H g)$} \\
\hline Control & $85 \pm 2$ & $84 \pm 2$ & $82 \pm 1$ & $83 \pm 1$ \\
\hline PV & $81 \pm 1$ & $82 \pm 1$ & $81 \pm 1$ & $83 \pm 1$ \\
\hline \multicolumn{5}{|c|}{$H R$ (beats $\mathrm{min}^{-1}$ ) } \\
\hline Control & $67 \pm 2$ & $64 \pm 2$ & $65 \pm 2$ & $64 \pm 2$ \\
\hline PV & $65 \pm 2$ & $63 \pm 2$ & $62 \pm 2$ & $63 \pm 2$ \\
\hline \multicolumn{5}{|c|}{ Aortic PWV $\left(m s^{-1}\right)$} \\
\hline Control & $8.3 \pm 0.9$ & $8.4 \pm 0.4$ & $8.3 \pm 0.3$ & $8.3 \pm 0.5$ \\
\hline PV & $8.7 \pm 0.5$ & $8.9 \pm 0.3$ & $8.5 \pm 0.4$ & $8.6 \pm 0.3$ \\
\hline
\end{tabular}

Abbreviations: DBP, diastolic blood pressure; HR, heart rate; MAP, mean arterial pressure; post-, minutes following 10 min of control and PV; PV, passive vibration; PWV, pulse wave velocity; SBP, systolic blood pressure. Values are means \pm s.e.

artery were calculated automatically. ${ }^{3,4} \mathrm{PWV}$ was calculated as distance/transit time. ${ }^{3}$ Measurements were collected before and $3 \mathrm{~min}$ (post-3), $15 \mathrm{~min}$ (post-15) and $30 \mathrm{~min}$ (post30) following PV or CON administration.

A $2 \times 3$ analysis of variance with repeated measures was conducted followed by Fisher's LSD test for pairwise comparisons. Pearson's correlation coefficient analysis was performed between changes in significant parameters. $P<0.05$ was considered to be significant.

There were no baseline differences between PV and CON, and no changes in BP, heart rate and aortic PWV after PV and CON (Table 1). At post-3 and post-15 after PV, baPWV decreased $(P<0.01)$ from baseline compared with CON $(P<0.01$, Figure 1a). At post-3 $(P<0.01)$, post-15 $(P<0.01)$ and

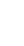

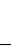



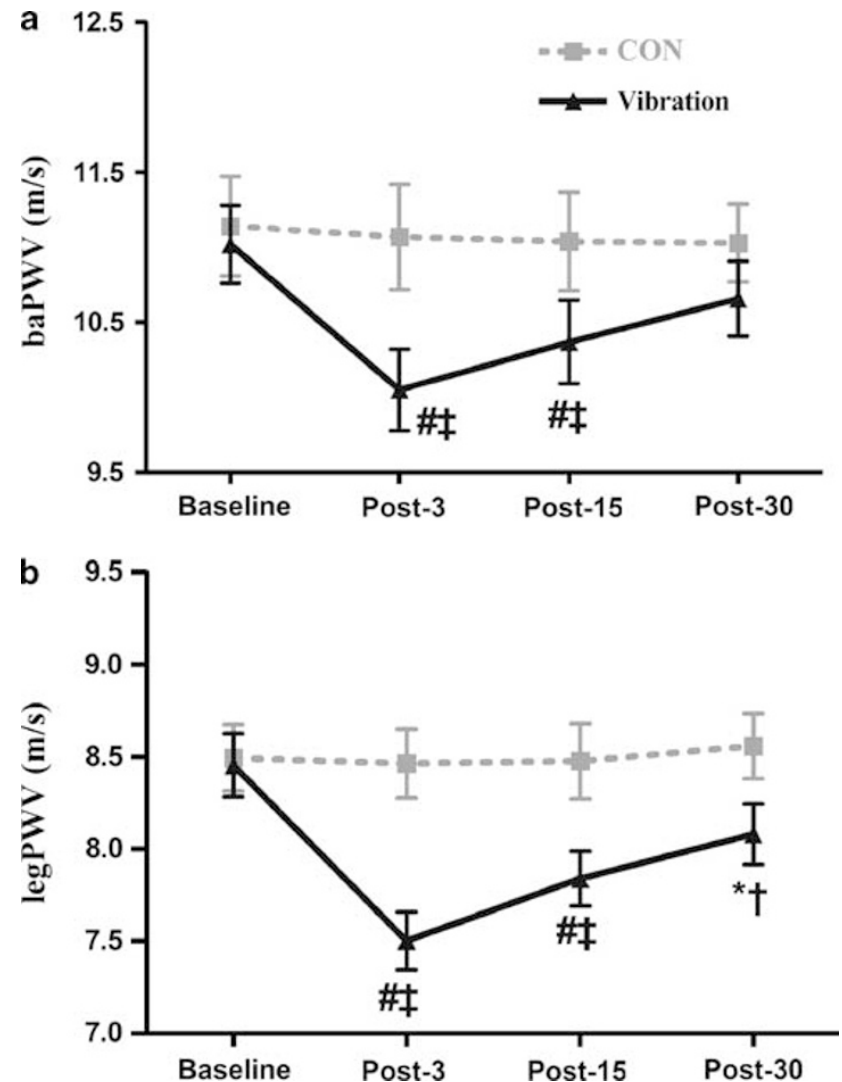

Figure 1 Brachial-ankle (baPWV) and leg (legPWV) pulse wave velocity at baseline, and 3,15 and $30 \mathrm{~min}$ following $10 \mathrm{~min}$ of control (CON) and passive vibration (PV) bouts. ${ }^{*} P<0.05$, ${ }^{\sharp} P<0.01$ vs. baseline. ${ }^{\dagger} P<0.05, \ddagger P<0.01$ treatment-by-time interaction. Values are means \pm s.e.

post-30 $(P<0.05)$ after PV, legPWV decreased from baseline compared with CON $(P<0.05$, Figure 1b). The decreases in legPWV and baPWV were correlated at post-3 $(r=0.81$, $P<0.01)$ and post-15 $(r=0.59, \quad P<0.01)$ after PV.

Our findings show a decrease in baPWV of $1.1 \mathrm{~m} \mathrm{~s}^{-1}$ after $10 \mathrm{~min}$ of continuous PV on the legs. This result is in agreement with that reported by Otsuki et al., ${ }^{1}$ who showed a decrease in baPWV within $40 \mathrm{~min}$ of ten 1-min sets of static squats with WBV. The magnitude of the baPWV decrease in Otsuki's study was smaller $\left(\sim 0.3 \mathrm{~m} \mathrm{~s}^{-1}\right)$ than the change observed in our study. This discrepancy could be due to the greater body area (whole legs vs. feet) that was directly and continuously exposed to vibration in our study (one 10-min set in our study vs. ten 1-min sets in Otsuki's study). As expected, aortic PWV was not altered after PV in this study, which is consistent with our previous findings showing that intermittent WBV does not reduce aortic PWV. ${ }^{2}$

We found that legPWV was significantly reduced throughout the 30-min recovery period after PV. Previous reports have noted a reduction in legPWV after acute leg exercise.,
Reductions in legPWV of $1 \mathrm{~ms}^{-1}$ or more have been shown after six sets of leg presses ${ }^{6}$ and $30 \mathrm{~min}$ of cycling exercise. ${ }^{5}$ Interestingly, the reduction in legPWV $\left(1.1 \mathrm{~m} \mathrm{~s}^{-1}\right)$ observed after PV in our study is greater than the reduction that we previously observed after ten 1-min sets of WBV. ${ }^{2}$ This reduction in peripheral PWV after PV could result from the direct and continuous application of vibration to the legs. In the present study, the decrease in legPWV was associated with a reduction in baPWV. As the main components of baPWV are aortic PWV and legPWV, it is likely that the reduction in baPWV noted after $\mathrm{PV}$ is attributable to the decrease in legPWV.

In our study, because there was no change in MAP, the decreases in PWV could be due to vasodilation of the muscular arteries. ${ }^{5} \mathrm{PV}$ has been shown to increase blood flow in the microvasculature of exposed limbs. ${ }^{10}$ Herrero et al. ${ }^{8}$ showed an increase in femoral artery blood flow after three 3-min sets of PV in patients with paraplegia. As the subjects were strapped to a table tilted at $45^{\circ}$ and PV was applied to the footboard, the short vasodilatory effect could result from the indirect application of PV to a small body area. This vasodilation may be related to an increase in nitric oxide production immediately after $\mathrm{PV}{ }^{10}$ As PV increases leg blood flow and neuromuscular activity, ${ }^{8} \mathrm{PV}$ might induce the production of vasodilatory metabolites. We can speculate that acute changes in legPWV after PV would be associated, in part, with endotheliummediated ${ }^{10}$ and contraction-related vasodilatory factors ${ }^{5}$ during early recovery and throughout the entire recovery period, respectively.

In conclusion, PV applied to the legs decreases legPWV and baPWV with no effect on aortic PWV and hemodynamics in young adults. Future studies are needed to investigate the acute- and long-term effects of PV on arterial function in individuals with increased $\mathrm{PWV}$, and the inability to perform conventional exercise.

\section{CONFLICT OF INTEREST}

The authors declare no conflict of interest.

Alexei Wong, Marcos A Sanchez-Gonzalez, Ryan Gil, Florence Vicil, Song Young Park and Arturo Figueroa

Department of Nutrition, Food and Exercise Sciences, The Florida State University, Tallahassee, FL, USA E-mail: afiguero@fsu.edu

1 Otsuki T, Takanami Y, Aoi W, Kawai Y, Ichikawa H, Yoshikawa T. Arterial stiffness acutely decreases after whole-body vibration in humans. Acta Physiol (Oxf) 2008; 194: 189-194.

2 Figueroa A, Vicil F, Sanchez-Gonzalez M. Acute exercise with whole-body vibration decreases wave reflection and leg arterial stiffness. Am J Cardiovasc Dis 2011; 1: 60-67.

3 Yamashina A, Tomiyama H, Takeda K, Tsuda H, Arai T, Hirose K, Koji Y, Hori S, Yamamoto Y. Validity, reproducibility, and clinical significance of noninvasive brachial-ankle pulse wave velocity measurement. Hypertens Res 2002; 25: 359-364.

4 Sugawara J, Hayashi K, Yokoi T, Cortez-Cooper MY, DeVan AE, Anton MA, Tanaka H. Brachial-ankle pulse wave velocity: an index of central arterial stiffness? J Hum Hypertens 2005; 19: 401-406.

5 Kingwell BA, Berry KL, Cameron JD, Jennings GL, Dart AM. Arterial compliance increases after moderateintensity cycling. Am J Physiol 1997; 273(5 Part 2): H2186-H2191.

6 Heffernan KS, Rossow L, Jae SY, Shokunbi HG, Gibson EM, Fernhall B. Effect of single-leg resistance exercise on regional arterial stiffness. Eur J Appl Physiol 2006; 98: 185-190.

7 Kerschan-Schindl K, Grampp S, Henk C, Resch H, Preisinger E, Fialka-Moser V, Imhof $\mathrm{H}$. Whole-body vibration exercise leads to alterations in muscle blood volume. Clin Physiol 2001; 21: 377-382.

8 Herrero AJ, Menendez H, Gil L, Martin J, Martin T, GarciaLopez D, Gil-Agudo A, Marin PJ. Effects of whole-body vibration on blood flow and neuromuscular activity in spinal cord injury. Spinal Cord 2011; 49: 554-559.

9 Maloney-Hinds C, Petrofsky JS, Zimmerman G. The effect of 30 vs $50 \mathrm{~Hz}$ passive vibration and duration of vibration on skin blood flow in the arm. Med Sci Monit 2008; 14: CR112-CR116.

10 Maloney-Hinds C, Petrofsky JS, Zimmerman G, Hessinger DA. The role of nitric oxide in skin blood flow increases due to vibration in healthy adults and adults with type 2 diabetes. Diabetes Technol Ther 2009; 11: 39-43. 